\title{
Cosmic Strings and the Origin of Globular Clusters
}

\author{
Alistair Barton, Robert H. Brandenberger and Ling Lin ${ }^{1, *}$ \\ ${ }^{1}$ Department of Physics, McGill University, Montréal, QC, H3A 2T8, Canada
}

\begin{abstract}
We hypothesize that cosmic string loops are the seeds about which globular clusters accrete. Fixing the cosmic string tension by demanding that the peak in the distribution of masses of objects accreting onto string loops agrees with the peak in the observed mass distribution of globular clusters in our Milky Way galaxy, we then compute the expected number density and mass function of globular clusters, and compare with observations. Our hypothesis naturally explains why globular clusters are the oldest and most dense objects in a galaxy, and why they are found in the halo of the galaxy.
\end{abstract}

\section{INTRODUCTION}

Globular clusters are spherical collections of stars which are found in the halos of galaxies which orbit the galactic center. Globular clusters are older and more dense than other star clusters found predominantly in the galactic disk. Our own Milky Way galaxy contains about 150 known globular clusters. Their mass distribution (shown in Fig. 1) has a peak at a mass of about $10^{5} M_{0}$, where $M_{0}$ is the solar mass. The origin of globular clusters is still not well understood (see e.g. 1] for a review).

In this Letter we propose that globular clusters are seeded by cosmic string loops. Cosmic strings exist in many particle physics models beyond the Standard Model (see 2 for reviews on cosmic strings and their role in cosmology). If Nature is described by a theory which admits cosmic string solutions, then a network of strings will inevitably form in the early universe and persist until the present time [4. The system of strings consists of a network of infinite strings with a typical curvature radius $\xi(t)$ which scales with the Hubble radius $H^{-1}(t)$, where $H$ is the Hubble expansion rate and $t$ is physical time, and a distribution of string loops. At any given time, the distribution of string loops is characterized by a critical radius $R_{c}(t)$ which dominates the mass function of string loops.

The cosmological consequences of cosmic strings are characterized by a single free parameters, namely the mass per unit length $\mu$ of the string (which is normally expressed in terms of the dimensionless number $G \mu$, where $G$ is Newton's gravitational constant). We will fix $G \mu$ by demanding that the mass which accretes about a loop with radius $R_{c}$ agrees with the observed peak mass in the globular cluster distribution. We then compute the predicted number density of such loops, and find that it agrees with the observed number density of globular clusters. We also compare the mass function of globular clusters predicted by our model with the data and obtain reasonable agreement. With one free parameter

*Electronic address: alistair.barton@mail.mcgill.ca, rhb@physics.mcgill.ca, ling.lin2@mail.mcgill.ca we have therefore fixed the peak mass, the overall number density and the mass function of globular clusters ${ }^{1}$. Note that the accretion of matter about string loops starts as soon as loops are created, i.e. much earlier than the time of reionization. Hence, our mechanism offers an explanation for why the globular clusters are the oldest components of a galaxy. The string loops are initially distributed throughout the region which eventually falls in to form the galaxy and will hence end up in the galactic halo rather than the disk.

Note that in our current analysis, we are neglecting both the initial translational velocities of the string loops as well as the "rocket effect", an anisotropic gravitational radiation reaction which can accelerate a string loop.

In the following, we first review the distribution of cosmic string loops resulting from any particle physics theory which gives rise to strings. In Section 3 we present our computations. We fix the one free parameter of the model, the string tension $G \mu$, by demanding that the objects accreting around string loops which dominate the loop mass function have the same mass as those corresponding to the peak in the mass distribution of globular clusters in the Milky Way. We then compute the predicted overall number density and the mass function of objects accreting about such cosmic string loops and compare with the observed mass distribution of globular clusters in our Milky Way galaxy. Section 4 presents our conclusions.

\section{COSMIC STRING LOOP DISTRIBUTION}

Cosmic strings are one-dimensional topological defects which arise in a large class of particle physics models beyond the Standard Model. They are lines of trapped energy density, and this energy gives rise to interesting signals in cosmology. The important point [4] is that if

\footnotetext{
1 There is a slight caveat to this statement: the predicted number density also depends on constants which are known from analytical considerations to be of the order 1 but whose exact values have to be determined by numerical cosmic string evolution simulations.
} 
Nature is described by a particle physics model admitting cosmic string solutions, then causality predicts that a network of strings will inevitably form in the early universe and will persist to the present time.

The energy scale $\eta$ of the new physics which yields cosmic strings determines the string mass per unit length $\mu$. In natural units the relation is $\mu=\eta^{2}$. The amplitude of the gravitational effects of the strings depends linearly on $\mu$. Strings come in two types: a network of infinite strings with a correlation length $\xi(t)$ which is proportional to the Hubble radius, and a distribution of string loops. Long string segments produce a conical discontinuity in space which leads to characteristic signals in CMB temperature maps [5, polarization maps [6] and $21 \mathrm{~cm}$ redshift maps [7] (see [8] for a recent overview of observational signals of long strings).

String loops can also play an important role in cosmology. In fact, in early studies of the role of strings in cosmology it was postulated [9] that strings could account for all of the observed structure. Specifically, it was postulated that galaxies in their entirety were seeded by strings. The one string - one galaxy hypothesis led to a value of the string tension $G \mu \sim 10^{-6}$, which corresponds to the energy scale $\eta$ of particle physics "Grand Unification". However, a cosmological model in which all fluctuations are due to strings leads to incoherent perturbations and predicts no acoustic oscillations in the CMB angular power spectrum [10. Thus, this model is ruled out by the $\mathrm{CMB}$ data [11. In fact, precision CMB observations such as ACT [12, SPT [13, WMAP [14] and Planck [15] lead to an upper bound on the string tension of 16 .

$$
G \mu<2 \times 10^{-7}
$$

(see 17] for older results). Tighter bounds can possibly be obtained by analyzing CMB maps in position space [18. Constraints on the amplitude of the stochastic background of gravitational waves produced by decaying cosmic string loops lead 19 to a stronger bound on $G \mu$ which, however, depends sensitively on the ratio of loop production radius to the Hubble radius (a number which the CMB bound is not sensitive to).

As the discussion of the previous paragraph has shown, cosmic strings cannot provide the dominant source of fluctuations. The dominant source must be due to processes in the early universe such as inflation [20] or alternatives (see 21] for a review of alternatives such as the "Matter Bounce" [22] or "String Gas Cosmology" [23]). Nevertheless, cosmic strings could have played an important role in cosmology, as long as the tension obeys the bound (1). For example, in many inflationary models cosmic strings will form at the end of inflation 24]. Furthermore, any phase transitions in matter which occur after the phase in the primordial universe which produces the dominant source of fluctuations (e.g. after the phase of inflation) will produce cosmic strings as long as the particle physics model admits string solutions. Hence, it is very interesting to search for the signals of strings in cosmology. If the effects are not seen to the level of a particular tension $\mu$, we will have ruled out all particle physics models yielding strings with symmetry breaking scale $\eta$ larger than $\sqrt{\mu}$. If strings are found, we can use them to explain cosmological phenomena which up to now are not explained. It is a particular application of the second possibility which we study in this Letter. But before explaining our ideas we must return to the review of the cosmic string scenario.

Cosmic string loops are typically generated by the intersection of segments of the long strings, segments which move through space at relativistic speeds. Causality tells us that the correlation length of the string network (which gives the average separation and average curvature radius of the long strings) is bounded from above by the Hubble radius. Dynamical arguments show that the correlation length at time $t$ cannot be parametrically smaller than $t$, otherwise string loops would be copiously produced and $\xi(t) / t$ would increase. The network of long strings thus takes on a "scaling solution" according to which the statistical distribution of strings is independent of time if all lengths are scaled to the Hubble radius.

The scaling distribution of strings implies that loops will be formed at any time $t$ with a typical radius $R_{f}(t)$ given by

$$
R_{f}(t)=\frac{\alpha}{\beta} t,
$$

where $\alpha$ is a constant which must be determined from numerical simulations. Recent simulations [25, 26] give $\alpha \sim 0.1$. The average length of a string loop is $l=\beta R$, where $\beta$ is a constant which would be $2 \pi$ if the strings were exactly circular. We shall use the value $\beta=10$.

Once formed, the number density of loops of radius $R$ redshifts because of the expansion of space. Loops also slowly decay by emitting gravitational radiation [27. Loops with radius smaller than

$$
R_{c}(t)=\gamma G \mu t
$$

will decay in less than a Hubble time. Here, $\gamma$ is another constant which is determined from numerical simulations. Its value is of order $\gamma \sim 10^{2}$. Combining these results, we find that the number density $n(R, t)$ of string loops of radius $R$ is given at times $t>t_{e q}$ (where $t_{e q}$ is the time of matter-radiation equality) by

$$
n(R, t) \sim t^{-2} R^{-2}
$$

for loops which form after $t_{e q}$ and

$$
\begin{aligned}
n(R, t) & =N \alpha^{5 / 2} \beta^{-5 / 2} t_{e q}^{1 / 2} t^{-2} R^{-5 / 2} \text { for } R>\gamma G \mu t \\
n(R, t) & =N \alpha^{5 / 2} \beta^{-5 / 2} t_{e q}^{1 / 2} \gamma^{-5 / 2}(G \mu)^{-5 / 2} t^{-9 / 2} \\
& =\text { const for } R<\gamma G \mu t
\end{aligned}
$$

for loops which form before $t_{e q}$. In the above, the constant $N$ depends on the average number $\tilde{N}$ of long string segments per Hubbe volume. Since two long string segments are required to form a loop, we could expect $N$ 
to depend on the square of $\tilde{N}$. We will be interested in values of $G \mu$ for which the critical radius (3) at the present time $t_{0}$ is smaller than $\alpha \beta^{-1} t_{e q}$. Such loops form before the time of equal matter and radiation, and their distribution is given by (5).

The total number density of strings is obtained by integrating (5) over the radius. The integral is dominated by $R \sim R_{c}(t)$. For times between $t_{e q}$ and the present time $t_{0}$ we obtain

$$
n_{\text {total }}(t) \simeq N \alpha^{5 / 2} \beta^{-5 / 2} \gamma^{-3 / 2} z_{\text {eq }}^{-3 / 4}(G \mu)^{-3 / 2} t^{-3},
$$

where $z(t)$ is the cosmological redshift at time $t$, and where the constant $5 / 3$ resulting from integrating over the loop distribution (5) has been absorbed in the factor $N$. Note, in particular, that the number density is larger for smaller values of $G \mu$. Hence, for such values of $G \mu$ there will be many string loops embedded in the region which collapses to form a galaxy (the galaxy seed being given not by cosmic strings, but by the dominant source of fluctuations). In fact, the accretion of matter about the galactic center sweeps up string loops from a larger region of space. Working in the Zel'dovich approximation 28], the local number density of string loops inside a galaxy will be enhanced by a factor of $F$. Accretion and virialization in each direction leads to the estimate $F=$ 64 .

In the following we will explore the possibility that the string loops are the seeds for the globular clusters embedded in the galactic halo.

\section{GLOBULAR CLUSTERS FROM COSMIC STRING LOOPS}

According to linear cosmological perturbation theory, accretion of matter about a cosmic string loop starts (modulo logarithmic growth at earlier times) at $t_{e q}$. At that time, the mass function of string loops is dominated by loops with radius

$$
R=\gamma G \mu t_{e q}
$$

Whereas these loops will have decayed by the present time, the objects they seed will keep growing. The local number density of such objects inside a galaxy is hence given by

$$
\begin{aligned}
n_{\text {local }} & =F N \alpha^{5 / 2} \beta^{-5 / 2} t_{e q}^{1 / 2} t_{0}^{-2} R_{c}\left(t_{e q}\right)^{-3 / 2} \\
& =F N \alpha^{5 / 2} \beta^{-5 / 2} \gamma^{-3 / 2} z_{e q}^{3 / 2}(G \mu)^{-3 / 2} t_{0}^{-3}
\end{aligned}
$$

The mass which has accreted about these seed loops at the present time ${ }^{2}$ is given by

$$
\begin{aligned}
M\left(\gamma G \mu t_{e q}, t_{0}\right) & =\beta \gamma(G \mu)^{2} z_{e q} \frac{t_{e q}}{G} \\
& =\beta \gamma(G \mu)^{2} z_{e q}^{-1 / 2} \frac{t_{0}}{G} .
\end{aligned}
$$

Inserting the values of $t_{0}$ and $G$ we find

$$
\frac{t_{0}}{G} \sim 10^{23} M_{0} .
$$

Making use of the values $\beta=10, \gamma=10^{2}$ and $z_{e q}=10^{4}$ and setting the mass (9) equal to the peak of the mass function of globular clusters in our galaxy $M_{G C} \sim 10^{5} M_{0}$ we find

$$
G \mu \sim 10^{-9.5} .
$$

Inserting this value into the local number density (8), using (in addition to the previously mentioned values for $\beta$ and $\gamma$ ) the values of the constants (see [26]) $\alpha=0.3$, $N=10^{2}$, and inserting the value of $t_{0}$ which yields $t_{0}^{-3} \sim$ $10^{-21} \mathrm{kpc}^{-3}$ we find

$$
n_{\text {local }} \sim 10^{-2}(k p c)^{-3} .
$$

This is of the same order of magnitude as the observed number density of globular clusters inside the Milky Way galaxy.

The cosmic string loop seed hypothesis for global clusters predicts the primordial mass function of globular clusters. The value of $M_{c}$, the peak in the mass function, was used in our analysis to fix the string tension. However, once the tension is fixed there is no more freedom in the mass distribution. For $M>M_{c}$ the mass function scales as $M^{-5 / 2}$ (as follows directly from the string loop distribution (5)). For masses smaller than $M_{c}$ we predict a linear decay. This follows from the fact that the loop radius distribution is flat but loops with radius smaller than $\gamma G \mu t$ live only a fraction of a Hubble time step which scales linearly with $R$.

In Fig. 1 we compare the predicted mass function (the solid line) with the observed distribution of globular clusters in the Milky Way (histogram values). The values for the blue histogram boxes come from the compilation in [3] of the properties of globular clusters in the Milky Way. The theoretical curve is obtained by taking the comoving number density $n\left(R, t_{e q}\right) z_{e q}^{3}$ of loops (where $n(R, t)$ is given in (5)), as a function of $R$, multiplying the result by the concentration factor $F$, allowing each loop to grow in mass by a factor of $z_{e q}$ (independent spherical accretion), and converting the distribution into a mass distribution $n\left(M, t_{0}\right)$, taking into account the Jacobian of the transformation from $R$ to $M$. The result is then multiplied

\footnotetext{
${ }^{2}$ We are here assuming that the accretion continues to the present time.
} 


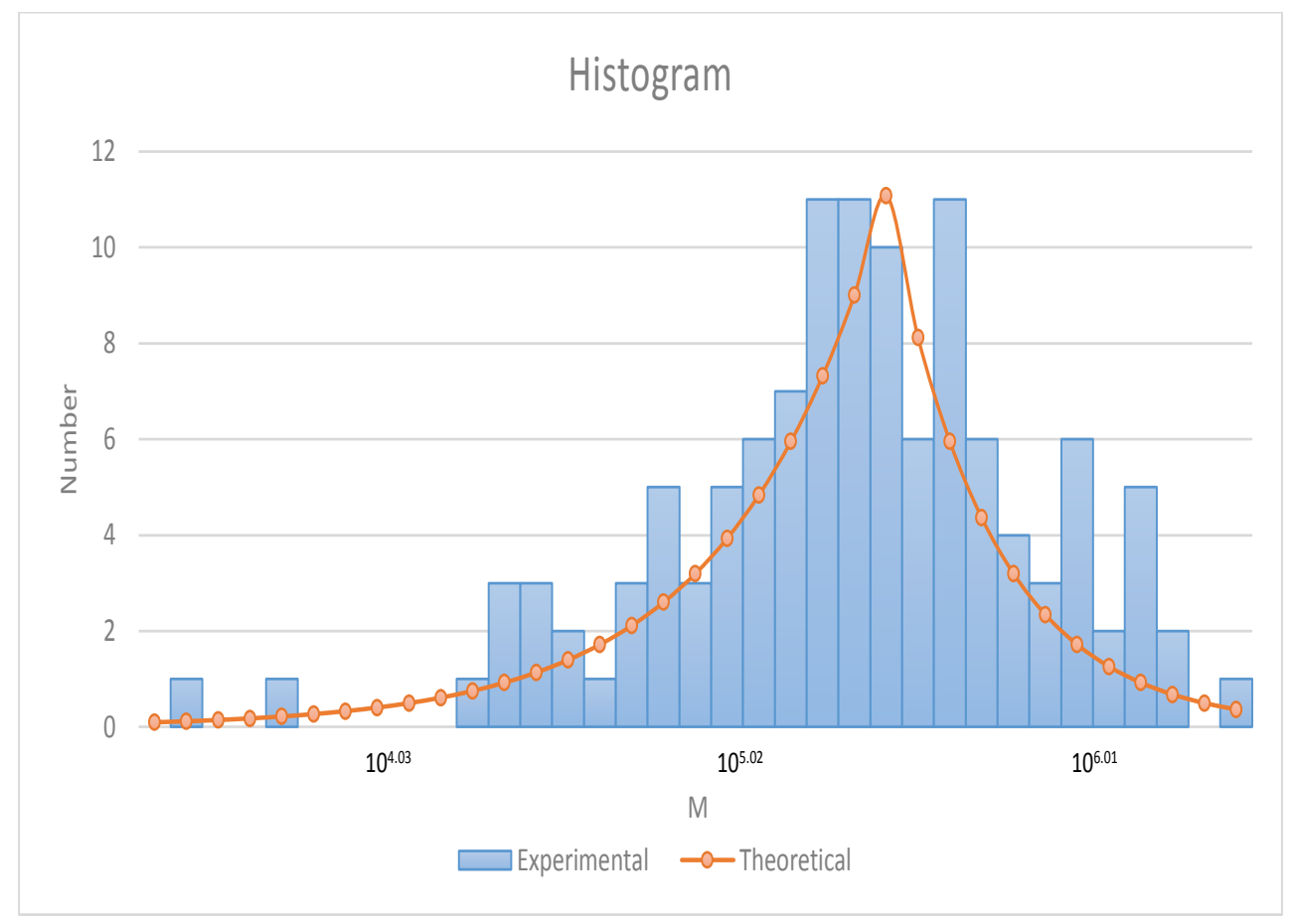

FIG. 1: Mass function of globular clusters in the Milky Way galaxy. The horizontal axis is mass on a logarithmic scale, the vertical axis gives the number density on a linear scale. The histogram show the data taken from 3. The solid curve is the prediction of our model for the value of $G \mu=5.43 \times 10^{-10}$ which minimizes $\chi^{2}$. The cosmic string parameters chosen are described in the text. Note, in particular, that the overall amplitude of the theoretical curve is predicted once $G \mu$ has been fixed to fit the peak location.

by the bin size $\delta M$ and by the volume of the Milky Way galaxy. Taking the bin size to be $\delta M=f M$, where $f$ is a number, we obtain for the peak number density bin

$$
\begin{aligned}
\delta N= & N F f \alpha^{5 / 2} \beta^{-5 / 2} \gamma^{-3 / 2} z_{e q}^{3 / 2} \\
& \times(G \mu)^{-3 / 2} V t_{0}^{-3}
\end{aligned}
$$

where $V$ is the volume of the galaxy. The peak mass of the theoretical curve is given by (9). In Fig. 1 the horizontal axis is mass (logarithmic scale) while the vertical axis gives the number of globular clusters (linear scale) inside of the Milky Way galaxy. The exact value of $G \mu$ for the theoretical prediction has been tuned to give the lowest value of $\chi^{2}$ between the theory curve and the data.

\section{CONCLUSIONS AND DISCUSSION}

We have seen that both the number density and mass of string loops with a mass per unit length given by (11)
Its value is $G \mu=5.43 \times 10^{-10}$.

In Fig. 2 we show how the peak position and amplitude of the mass function shift as $G \mu$ varies. As $G \mu$ decreases, the peak mass decreases but the predicted number of globular clusters increases. The surprising result is that a good fit of both amplitude and peak number density occur for the same value of $G \mu^{3}$.

Our analysis does not take into account interactions between globular clusters and other processes which can change the mass of a globular cluster as a function of time during the highly nonlinear process of galaxy formation. Another effect which we have neglected in the current analysis is that of initial translational motion of string loops.

is correct to explain the origin of globular clusters. We hypothesize that globular clusters are seeded by string loops which dominate the loop mass distribution at the 


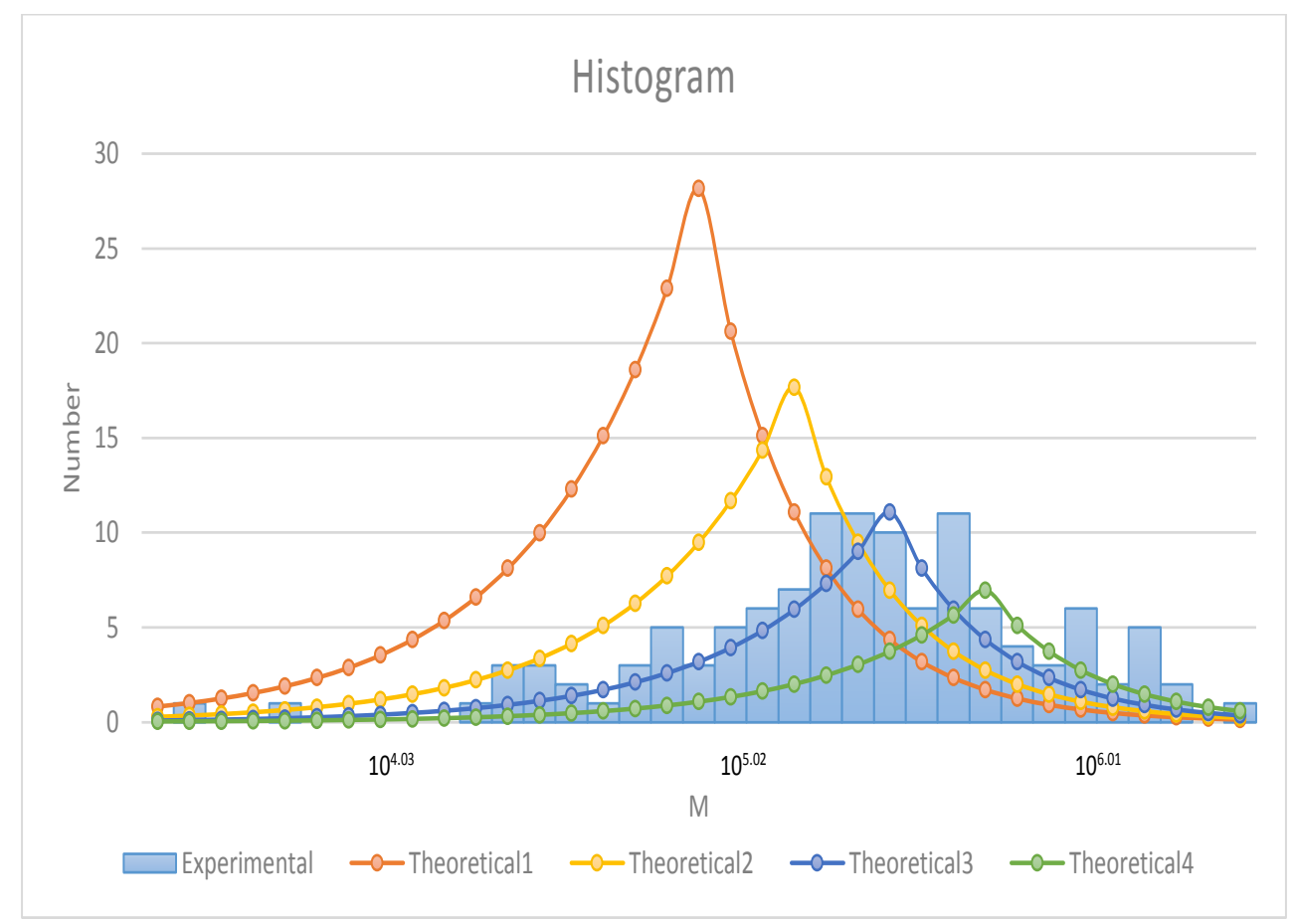

FIG. 2: Dependence of the mass function of our model on the string tension $G \mu$. The curves shown are for $G \mu=2.92 \times$ $10^{-10}, G \mu=3.98 \times 10^{-10}, G \mu=5.43 \times 10^{-10}$ and $G \mu=7.41 \times 10^{-10}$ (in increasing order of mass at the peak position). The axes and data are the same as in the previous figure.

time $t_{e q}$ when the accretion of cold matter onto the loops can start. We emphasize that fixing one free parameter has allowed us to fit both the mass and the number density of globular clusters. In addition, the model predicts a mass function which is in reasonable agreement with observations. Our model for the origin of globular clusters also naturally explains the fact that the globular clusters are the oldest star clusters in a galaxy, that they are the densest regions, and that they are distributed in the galactic halo rather than confined to the galactic disk.

Our theory predicts the the number of globular clusters in a galaxy will scale linearly with the mass of the galaxy. It also predicts that the mass function of globular clusters should be universal across galaxies. Since the energy distribution of dark matter falling onto a cosmic string is more peaked than that falling into a Gaussian fluctuation 29], we predict falling velocity rotation curves about the center of a globular cluster. Not all string loops are swept up into galaxies. Hence, our model also predicts the presence of compact objects of globular cluster mass in the field between galaxies. The corresponding number density does not include the factor $F \sim 10^{2}$.

In the current analysis we have neglected the initial translational motion of the string loops. Since this peculiar velocity redshifts and since we are considering string loops produced long before the time of equal matter and radiation, the loop motion should be a small effect by the time that the loop starts accreting matter at $t_{e q}$. We are currently in the process of working out the details. An effect which might be more important (and which we are neglecting here) is the rocket effect.

A question which we have not addressed here is the issue of star formation inside the object which accretes onto the string loops (see e.g. 30, 31 for recent studies of this question in the context of early structure formation from strings). There may well be a significant difference in the star formation processes for matter accreting onto a string loop inside a galaxy versus in the field.

\section{Acknowledgments}

We wish to thank G. Holder, Y. Omori, T. Webb and S. Yamanouchi for valuable discussions. RB is supported by an NSERC Discovery Grant, and by funds from the 
Canada Research Chair program.

[1] J. P. Brodie and J. Strader, "Extragalactic globular clusters and galaxy formation," Ann. Rev. Astron. Astrophys. 44, 193 (2006) astro-ph/0602601;

W. E. Harris, "Massive star clusters in galaxies," Trans. Roy. Soc. Lond. 368, 889 (2010) arXiv:0911.0798 [astroph.GA]].

[2] A. Vilenkin and E.P.S. Shellard, Cosmic Strings and other Topological Defects (Cambridge Univ. Press, Cambridge, 1994);

M. B. Hindmarsh and T. W. B. Kibble, "Cosmic strings," Rept. Prog. Phys. 58, 477 (1995) arXiv:hep$\mathrm{ph} / 9411342$;

R. H. Brandenberger, "Topological defects and structure formation," Int. J. Mod. Phys. A 9, 2117 (1994) arXiv:astro-ph/9310041.

[3] O. Y. Gnedin and J. P. Ostriker, "Destruction of the galactic globular cluster system," Astrophys. J. 474, 223 (1997) astro-ph/9603042.

[4] T. W. B. Kibble, "Phase Transitions In The Early Universe," Acta Phys. Polon. B 13, 723 (1982);

T. W. B. Kibble, "Some Implications Of A Cosmological Phase Transition," Phys. Rept. 67, 183 (1980).

[5] N. Kaiser and A. Stebbins, "Microwave Anisotropy Due To Cosmic Strings," Nature 310, 391 (1984).

[6] R. J. Danos, R. H. Brandenberger and G. Holder, "A Signature of Cosmic Strings Wakes in the CMB Polarization," Phys. Rev. D 82, 023513 (2010) arXiv:1003.0905 [astro-ph.CO]].

[7] R. H. Brandenberger, R. J. Danos, O. F. Hernandez and G. P. Holder, "The $21 \mathrm{~cm}$ Signature of Cosmic String Wakes," JCAP 1012, 028 (2010) arXiv:1006.2514 [astroph.CO]].

[8] R. H. Brandenberger, "Searching for Cosmic Strings in New Observational Windows," Nucl. Phys. Proc. Suppl. 246-247, 45 (2014) arXiv:1301.2856 [astro-ph.CO]].

[9] N. Turok and R. H. Brandenberger, "Cosmic Strings And The Formation Of Galaxies And Clusters Of Galaxies," Phys. Rev. D 33, 2175 (1986);

H. Sato, "Galaxy Formation by Cosmic Strings," Prog. Theor. Phys. 75, 1342 (1986);

A. Stebbins, "Cosmic Strings and Cold Matter", Ap. J. (Lett.) 303, L21 (1986).

[10] J. Magueijo, A. Albrecht, D. Coulson and P. Ferreira, "Doppler peaks from active perturbations," Phys. Rev. Lett. 76, 2617 (1996) arXiv:astro-ph/9511042;

U. L. Pen, U. Seljak and N. Turok, "Power spectra in global defect theories of cosmic structure formation," Phys. Rev. Lett. 79, 1611 (1997) arXiv:astro$\mathrm{ph} / 9704165$.

[11] P. D. Mauskopf et al. [Boomerang Collaboration], "Measurement of a Peak in the Cosmic Microwave Background Power Spectrum from the North American test flight of BOOMERANG," Astrophys. J. 536, L59 (2000) arXiv:astro-ph/9911444.

[12] A. Kosowsky [the ACT Collaboration], "The Atacama Cosmology Telescope Project: A Progress Report," New Astron. Rev. 50, 969 (2006) arXiv:astro-ph/0608549. J. L. Sievers et al. [Atacama Cosmology Telescope Col- laboration], "The Atacama Cosmology Telescope: Cosmological parameters from three seasons of data," JCAP 1310, 060 (2013) arXiv:1301.0824 [astro-ph.CO]].

[13] J. E. Ruhl et al. [The SPT Collaboration], "The South Pole Telescope," Proc. SPIE Int. Soc. Opt. Eng. 5498, 11 (2004) arXiv:astro-ph/0411122;

Z. Hou, C. L. Reichardt, K. T. Story, B. Follin, R. Keisler, K. A. Aird, B. A. Benson and L. E. Bleem et al., "Constraints on Cosmology from the Cosmic Microwave Background Power Spectrum of the $2500 \mathrm{deg}^{2}$ SPT-SZ Survey," Astrophys. J. 782, no. 2, 74 (2014) arXiv:1212.6267 [astro-ph.CO]].

[14] C. L. Bennett et al., "First Year Wilkinson Microwave Anisotropy Probe (WMAP) Observations: Preliminary Maps and Basic Results," Astrophys. J. Suppl. 148, 1 (2003) arXiv:astro-ph/0302207.

[15] P. A. R. Ade et al. [Planck Collaboration], "Planck 2013 results. XVI. Cosmological parameters," Astron. Astrophys. 571, A16 (2014) arXiv:1303.5076 [astro-ph.CO]].

[16] C. Dvorkin, M. Wyman and W. Hu, "Cosmic String constraints from WMAP and the South Pole Telescope," Phys. Rev. D 84, 123519 (2011) arXiv:1109.4947 [astroph.CO]];

P. A. R. Ade et al. [Planck Collaboration], "Planck 2013 results. XXV. Searches for cosmic strings and other topological defects," Astron. Astrophys. 571, A25 (2014) arXiv:1303.5085 [astro-ph.CO]].

[17] L. Pogosian, S. H. H. Tye, I. Wasserman and M. Wyman, "Observational constraints on cosmic string production during brane inflation," Phys. Rev. D 68, 023506 (2003) [Erratum-ibid. D 73, 089904 (2006)] arXiv:hepth/0304188;

M. Wyman, L. Pogosian and I. Wasserman, "Bounds on cosmic strings from WMAP and SDSS," Phys. Rev. D 72, 023513 (2005) [Erratum-ibid. D 73, 089905 (2006)] arXiv:astro-ph/0503364;

A. A. Fraisse, "Limits on Defects Formation and Hybrid Inflationary Models with Three-Year WMAP Observations," JCAP 0703, 008 (2007) arXiv:astroph/0603589;

U. Seljak, A. Slosar and P. McDonald, "Cosmological parameters from combining the Lyman-alpha forest with CMB, galaxy clustering and SN constraints," JCAP 0610, 014 (2006) arXiv:astro-ph/0604335;

R. A. Battye, B. Garbrecht and A. Moss, "Constraints on supersymmetric models of hybrid inflation," JCAP 0609, 007 (2006) arXiv:astro-ph/0607339;

R. A. Battye, B. Garbrecht, A. Moss and H. Stoica, "Constraints on Brane Inflation and Cosmic Strings," JCAP 0801, 020 (2008) arXiv:0710.1541 [astro-ph]];

N. Bevis, M. Hindmarsh, M. Kunz and J. Urrestilla, "CMB power spectrum contribution from cosmic strings using field-evolution simulations of the Abelian Higgs model," Phys. Rev. D 75, 065015 (2007) arXiv:astro$\mathrm{ph} / 0605018$;

N. Bevis, M. Hindmarsh, M. Kunz and J. Urrestilla, "Fitting CMB data with cosmic strings and inflation," Phys. Rev. Lett. 100, 021301 (2008) astro-ph/0702223 
[ASTRO-PH]];

R. Battye and A. Moss, "Updated constraints on the cosmic string tension," Phys. Rev. D 82, 023521 (2010) arXiv:1005.0479 [astro-ph.CO]].

[18] R. J. Danos and R. H. Brandenberger, "Canny Algorithm, Cosmic Strings and the Cosmic Microwave Background," Int. J. Mod. Phys. D 19, 183 (2010) arXiv:0811.2004 [astro-ph]];

S. Amsel, J. Berger and R. H. Brandenberger, "Detecting Cosmic Strings in the CMB with the Canny Algorithm," JCAP 0804, 015 (2008) arXiv:0709.0982 [astro-ph]];

A. Stewart and R. Brandenberger, "Edge Detection, Cosmic Strings and the South Pole Telescope," JCAP 0902, 009 (2009) arXiv:0809.0865 [astro-ph]].

[19] R. Battye and A. Moss, "Updated constraints on the cosmic string tension," Phys. Rev. D 82, 023521 (2010) arXiv:1005.0479 [astro-ph.CO]].

[20] Guth AH, "The Inflationary Universe: A Possible Solution To The Horizon And Flatness Problems," Phys. Rev. D 23, 347 (1981);

R. Brout, F. Englert and E. Gunzig, "The Creation Of The Universe As A Quantum Phenomenon," Annals Phys. 115, 78 (1978);

A. A. Starobinsky, "A New Type Of Isotropic Cosmological Models Without Singularity," Phys. Lett. B 91, 99 (1980);

K. Sato, "First Order Phase Transition Of A Vacuum And Expansion Of The Universe," Mon. Not. Roy. Astron. Soc. 195, 467 (1981).

[21] R. H. Brandenberger, "Alternatives to the inflationary paradigm of structure formation," Int. J. Mod. Phys. Conf. Ser. 01, 67 (2011) arXiv:0902.4731 [hep-th]].

[22] D. Wands, "Duality invariance of cosmological perturbation spectra," Phys. Rev. D 60, 023507 (1999) arXiv:grqc/9809062;

F. Finelli and R. Brandenberger, "On the generation of a scale-invariant spectrum of adiabatic fluctuations in cosmological models with a contracting phase," Phys. Rev. D 65, 103522 (2002) arXiv:hep-th/0112249.

[23] R. H. Brandenberger and C. Vafa, "Superstrings In The Early Universe," Nucl. Phys. B 316, 391 (1989).;

A. Nayeri, R. H. Brandenberger and C. Vafa, "Producing a scale-invariant spectrum of perturbations in a Hagedorn phase of string cosmology," Phys. Rev. Lett. 97, 021302 (2006) arXiv:hep-th/0511140;

R. H. Brandenberger, A. Nayeri, S. P. Patil and C. Vafa, "String gas cosmology and structure formation," Int. J. Mod. Phys. A 22, 3621 (2007) hep-th/0608121;

R. H. Brandenberger, "String Gas Cosmology," arXiv:0808.0746 [hep-th].

[24] R. Jeannerot, "A Supersymmetric SO(10) Model with Inflation and Cosmic Strings," Phys. Rev. D 53, 5426 (1996) arXiv:hep-ph/9509365;
R. Jeannerot, J. Rocher and M. Sakellariadou, "How generic is cosmic string formation in SUSY GUTs," Phys. Rev. D 68, 103514 (2003) arXiv:hep-ph/0308134;

S. Sarangi and S. H. H. Tye, "Cosmic string production towards the end of brane inflation," Phys. Lett. B 536, 185 (2002) arXiv:hep-th/0204074.

[25] A. Albrecht and N. Turok, "Evolution Of Cosmic Strings," Phys. Rev. Lett. 54, 1868 (1985);

D. P. Bennett and F. R. Bouchet, "Evidence For A Scaling Solution In Cosmic String Evolution," Phys. Rev. Lett. 60, 257 (1988);

B. Allen and E. P. S. Shellard, "Cosmic String Evolution: A Numerical Simulation," Phys. Rev. Lett. 64, 119 (1990);

C. Ringeval, M. Sakellariadou and F. Bouchet, "Cosmological evolution of cosmic string loops," JCAP 0702, 023 (2007) arXiv:astro-ph/0511646;

V. Vanchurin, K. D. Olum and A. Vilenkin, "Scaling of cosmic string loops," Phys. Rev. D 74, 063527 (2006) arXiv:gr-qc/0511159.

[26] J. J. Blanco-Pillado, K. D. Olum and B. Shlaer, "Large parallel cosmic string simulations: New results on loop production," Phys. Rev. D 83, 083514 (2011) arXiv:1101.5173 [astro-ph.CO]];

L. Lorenz, C. Ringeval and M. Sakellariadou, "Cosmic string loop distribution on all length scales and at any redshift," JCAP 1010, 003 (2010) arXiv:1006.0931 [astro-ph.CO]];

J. J. Blanco-Pillado, K. D. Olum and B. Shlaer, "The number of cosmic string loops," Phys. Rev. D 89, no. 2, 023512 (2014) arXiv:1309.6637 [astro-ph.CO]].

[27] T. Vachaspati and A. Vilenkin, "Gravitational Radiation from Cosmic Strings," Phys. Rev. D 31, 3052 (1985); R. L. Davis, "Nucleosynthesis Problems for String Models of Galaxy Formation", Phys. Lett. B 161, 285 (1985).

[28] Y. .B. Zeldovich, "Gravitational instability: An Approximate theory for large density perturbations," Astron. Astrophys. 5, 84 (1970).

[29] R. H. Brandenberger, N. Kaiser, D. N. Schramm and N. Turok, "Galaxy and Structure Formation with Hot Dark Matter and Cosmic Strings," Phys. Rev. Lett. 59, 2371 (1987);

R. H. Brandenberger, N. Kaiser and N. Turok, "Dissipationless Clustering of Neutrinos Around a Cosmic String Loop," Phys. Rev. D 36, 2242 (1987).

[30] B. Shlaer, A. Vilenkin and A. Loeb, "Early structure formation from cosmic string loops," JCAP 1205, 026 (2012) arXiv:1202.1346 [astro-ph.CO]].

[31] F. Duplessis and R. Brandenberger, "Note on Structure Formation from Cosmic String Wakes," JCAP 1304, 045 (2013) arXiv:1302.3467 [astro-ph.CO]]. 